\title{
Value of surveillance cultures in a bone marrow transplantation unit
}

\author{
ÉVA CZIRÓK, G. Y. PRINZ*, R. DÉNES†, P. REMÉNYI† and AGNES HERENDI \\ B. Johan National Institute of Public Health, Gyáli u 2-4, H-1097 Budapest, *Szent László Hospital, Gyáli u 5-7, \\ H-1097 Budapest and ${ }^{\dagger}$ National Institute of Haematology, Blood Transfusion, Transplantation and Immunology, \\ Daróczi u24, H-1113 Budapest, Hungary
}

\begin{abstract}
Because of the increased risk of infection with the associated diagnostic and therapeutic problems in bone marrow transplantation (BMT) patients, the usefulness of surveillance cultures (SC) at the BMT department of the National Institute of Haematology, Blood Transfusion, Transplantation and Immunology, Budapest, was reviewed. Between January 1992 and May 1995, 26 BMT operations were performed; 13 patients had 23 febrile espisodes. In 12 of these episodes infection was clinically documented; however, SC of these patients yielded bacteria identical with those in the blood culture in only two episodes ( 1 and 6 days before their blood cultures became positive, respectively). Out of a total of 1187 samples from these patients, potentially pathogenic bacteria were isolated from $145 \mathrm{SC}$ and 43 blood cultures (drawn on 31 different days). Suppression of the gastrointestinal flora could be achieved by the department's decontamination regimen; however, overgrowth by gram-positive organisms (mainly coagulase-negative staphylococci) occurred in the intestine and at other body sites. On the basis of these results, $\mathrm{SC}$ are of limited value in predicting infection or identifying the causative organisms of fever. On the other hand, SC are useful in confirming the efficiency of suppression of the body flora by antimicrobial agents. Specific treatment was based on suitably sampled materials, and close contact between physicians, infectious disease specialists and microbiologists was essential.
\end{abstract}

\section{Introduction}

All organ transplant patients require immunosuppression, which can produce a wide range of undesirable side-effects and in particular, a predisposition to serious infections. The extreme vulnerability of these patients has led to the institution of various anti-infective measures.

Infection control in a bone marrow transplantation (BMT) department is complex [1]. Environmental exposures should be minimised. Patient hygiene, the use of masks by the staff, strict enforcement of handwashing by staff and visitors and the isolation of transplant recipients are beneficial [1]. Decontamination of water supplies and foods and filtering air (frequently by using laminar air flow) may also decrease the incidence of serious bacterial and fungal infections [2]. Antimicrobial prophylaxis against opportunist pathogens, selective decontamination of

Received 16 April 1996; revised version accepted 13 Feb. 1997.

Corresponding author: Dr E. Czirók. intestinal bacterial flora with oral non-absorbable antibiotics [2], as well as fungal prophylaxis, also minimise post-operative complications. Surveillance cultures (SC) are used as additional measures $[3,4]$, because most infections in these patients are caused by organisms from the patient's own microbial flora.

As the value of $\mathrm{SC}$ in routine management remains controversial $[5,6]$, this study aimed to analyse the value of $\mathrm{SC}$ in a bone marrow transplantation unit with particular reference to bacteriological results.

\section{Materials and methods}

\section{Surveillance specimens}

Specimens were taken from nose, throat, skin, catheter, faeces, vagina or foreskin, and urine, immediately after intestinal decontamination had started, and after transplantation. Collection and processing were carried out twice weekly, while other cultures (sputum, blood, etc.) were taken as clinically indicated. In the event of fever, three sets of blood cultures were drawn from each lumen of the Hickman catheter and from a peripheral vein. Oxoid 'Signal' media were used for blood 
cultures. The specimens were cultured for grampositive and gram-negative aerobic bacteria, which were defined as potential pathogens. Cultures were analysed only qualitatively. Bacterial identification and antimicrobial susceptibility tests were performed according to Hungarian standard methods [7].

\section{Antibacterial prevention measures}

The aerobic component of normal flora was suppressed according to the following regimen. In the course of the preparation of the patient chlorhexidine and amphotericin B for the oral cavity, PVP iodine for skin; mupirocin or gentamicin and clotrimazole for the skin and for vagina or foreskin; tobramycin for eye, ear and nose; amphotericin-B and vancomycin for catheters were employed, all being applied locally. From the 11th day before transplantation, decontamination of the gut flora and an antibiotic prevention regimen were employed according to the following protocol: ciprofloxacin, metronidazole and miconazole, tobramycin, and, if Staphylococcus occurred in faeces, vancomycin were given in standard dosage. For patients with neutrophil counts below 1000, prophylaxis and nursing in a sterile environment were obligatory. In initial febrile episodes a combination of ceftazidime and amikacin, or alternatively piperacillin or imipenem and netilmicin was employed. If fever did not cease, vancomycin, or alternatively teicoplanin was introduced to broaden the cover, followed by amphotericin B 3 days later if fever continued. Besides empirical therapy, appropriate antimicrobial agents were employed if a causative agent was detected.

\section{Patients}

A total of 26 BMT were carried out in the BMT Department of the National Institute of Blood Transfusion and Immunology between 1 January 1992 and 15 May 1995. The clinical and microbiological records of all 26 patients were reviewed and evaluated. Episodes in which a patient had fever were recorded. In particular, those patients whose specimens were taken during the first 30 days of the post-transplantation period, i.e., the time when neutropenia was maximal, were included in the analysis of SC. Thus the results of SC of early infection complications were analysed primarily.

\section{Results}

\section{Culture results of blood and surveillance cultures} compared with other data

Thirteen of 26 transplant patients had febrile episodes in the course of which an infectious origin of the fever was suspected (Table 1). Fourteen of the 23 febrile episodes in these 13 patients yielded positive blood cultures. In 12 of 23 febrile episodes clinically documented infection could be detected. In two of these episodes (1/1 and 12/3) the supposed causative agents were viral, and in three fungal $(7 / 1,9 / 2,13 / 1)$. Bacterial infection was documented in blood cultures in four patients $(3 / 1,4 / 1,5 / 1,10 / 1)$. SC from these patients yielded an identical organism to that of the blood culture in only one episode (10/1). Sepsis in this patient was due to serious cytostatic treatment causing mucositis of the gastrointestinal tract, thus allowing bacterial invasion of the bowel wall. In this case colonisation was demonstrated 1 day earlier than the blood culture. In two episodes $(6 / 2,12 / 2)$ the infectious origin of fever could not be verified microbiologically. In one episode (11/1) blood cultures were positive but the fever was explained by graft versus host disease (GVHD); and in one episode (2/1) catheter-associated infection was considered to be the cause of the fever. In three episodes $(6 / 1,9 / 1,12 / 1)$ fever occurred during the conditioning regimen, but an infectious cause could be excluded in these cases. There were five further episodes $(2 / 2,3 / 2,4 / 2,12 / 4,13 / 2)$ in the course of which fever occurred after the 30th post-transplantation day (i.e., late infection complication). Sepsis developed because of acute GVHD with intestinal manifestation in two of these episodes $(3 / 2,12 / 4)$ rendering the patient susceptible to infection. In a later case an SC yielded bacteria identical to the blood isolates and it was positive 7 days before the blood culture became positive. In one case (8/1) apoplexy occurred with febrile symptoms. In another case $(5 / 2)$ fever ceased when granulocytes re-appeared.

Apart from viral infections $(1 / 1,12 / 3)$, in five of 23 episodes bacteria $(3 / 1,5 / 1,10 / 1,12 / 4)$ and fungi $(7 / 1)$ were isolated from blood cultures, verifying the causative agent of infection. In all the other 16 episodes - of which nine blood cultures were positive - the causative agent of microbiological infection could not be verified with certainty, because either blood or SC were negative or blood revealed merely a potential pathogen $(3 / 2,4 / 1)$, and did not unambiguously prove the aetiological agent of the infection. The aetiological role of potentially pathogenic bacteria isolated from blood was supported by the organism being repeatedly isolated from blood culture or its isolation from other body sites.

Of 1187 samples from 13 patients (except blood cultures), potential pathogens were isolated from 145 SC. One species of a potential pathogen was present in three patients $(4 / 1,12 / 2,13 / 2)$, and two were present in five $(3 / 2,5 / 1,5 / 2,6 / 1,12 / 1)$ (Table 1). Of the 5 remaining patients, bacteria could not be detected in five SC while on seven occasions threeto-five kinds of bacteria were detected. Three patients were found to be colonised by as many as seven $(2 / 1)$, eight $(2 / 2)$ and $12(6 / 2)$ different kinds of bacteria, respectively, at different sites of their body in the course of one episode. For example, coagulasenegative staphylococci (CNS), Pseudomonas aeruginosa, Staphylococcus aureus, two different enterobac- 
teria, two different streptococci and Enterococcus spp., Corynebacterium spp. and Mycoplasma pneumoniae were all isolated from the SC of patient no. 6 .

\section{Colonisation of different body sites of decontaminated patients}

The most common sites of colonisation (Table 2) were the skin (31 positive results from 136 samples), throat (29 of 192) and foreskin or vagina (32 of 126). The outside of venous catheters was positive in 3 of 338 instances. Gram-positive bacteria made up the majority of strains isolated from all sites (91 gram-positive versus 41 gram-negative) with the exception of throat and urine samples, where the gram-negative isolates outnumbered the gram-positive isolates. A predominance of gram-positive isolates was more clearly expressed in blood cultures (13 versus 4).

Of the gram-positive bacteria, CNS (62 strains) were by far the most commonly isolated from all sites of the body. Moreover, enterococci frequently colonised the skin (two isolates) and foreskin or vagina (eight) and intestine (seven). Colonisation with $P$. aeruginosa was also found (four isolates originated from the urine of three patients, and another nine isolates colonised the throat and vagina of the third patient). Among other gram-negative isolates, Enterobacteriaceae occurred in SC, mainly in the throat (14 of 28) (Table 2).

In faecal samples (Table 2) E. cloacae could be isolated after transplantation (three isolates from 80 samples) but Enterococcus spp. and CNS were more common (seven isolates of each) during the first 30 days. $P$. aeruginosa also occurred in faeces. Clostridium difficile was common among these patients, mainly before transplantation and several months after transplantation (data not shown).

\section{Incidence of different potentially pathogenic bacteria in surveillance cultures}

It can be seen from Table 2 that CNS were the most frequently cultured gram-positive bacteria (62 isolates) while Enterococcus spp. (18) and Streptococcus spp. (9), occurred frequently. P. aeruginosa (14) and klebsiellae (26), were isolated frequently among gram-negative strains. CNS also occurred most frequently in blood cultures (nine instances), followed by Str. mitis (2), and enterococci (2). Among gramnegative organisms, $P$. aeruginosa was also isolated from blood.

\section{Discussion}

In bone marrow transplantation the chance of infection is high, as the early post-transplantation period is characterised by neutropenia; moreover, chemotherapy and total body irradiation, as well as the use of venous catheters, contribute to the breakdown of normal anatomical barriers. This chronic risk of infection with its diagnostic problems and potentially fatal outcome requires an understanding of the principles of transplant-associated infections. Because controversial results from SC have been described in the literature $[8,9]$ the usefulness of $\mathrm{SC}$ was reviewed in this Department.

No correlation was observed between positive SC and clinically documented infections, because only in two of 23 febrile episodes were potential pathogens predicted by $\mathrm{SC}$, while in several other cases, results of positive cultures merely suggested a possible cause of infection. On the one hand, colonisation was frequent, but on the other hand, documented infections with potential pathogens were not. Moreover, SC were frequently negative in clinically documented infections so that the predictive value of SC is low. The febrile transplant patient should be evaluated only with blood cultures.

The demonstration that 12 of 26 transplantation patients had microbiologically documented infections is in agreement with the study by Atkinson et al. [2], as they also found that bacteraemia occurred in at least one-half of all patients. The findings of the present study show SC to be unhelpful as a predictor of bacteraemia and agree with those of Milligan and Taylor [10], who also noted that gram-negative bacteria were responsible in only a few cases of bacteriaemia.

The results of this study show that SC are of limited value in predicting infection or identifying the causative organism of fever. Thus, in the future it will be sufficient in this Department to obtain SC only once a week until the marrow graft has taken successfully.

In the light of the low predictive value that this and other studies have shown the question may be raised as to whether SC are of any use. However, SC in BMT patients have been useful in predicting the efficiency of suppression by antimicrobial agents of body flora whose component organisms often lead to invasive infection in neutropenic patients.

As far as the efficiency of decontamination is concerned, the regimen used in this study successfully suppressed the gastrointestinal flora. However, overgrowth by gram-positive organisms, mainly CNS, could occur. Antimicrobial agents suppressed the aerobic faecal flora and the elimination rate for the so-called normal flora, e.g., Escherichia coli was greater than for klebsiellae, multi-resistant enterococci, pseudomonads or Candida spp. An interesting observation was the re-appearance of bacterial species in the bowel. Even 2-5 months after decontamination different species of enterobacteria and streptococci appeared in the gut in pure culture or in large 


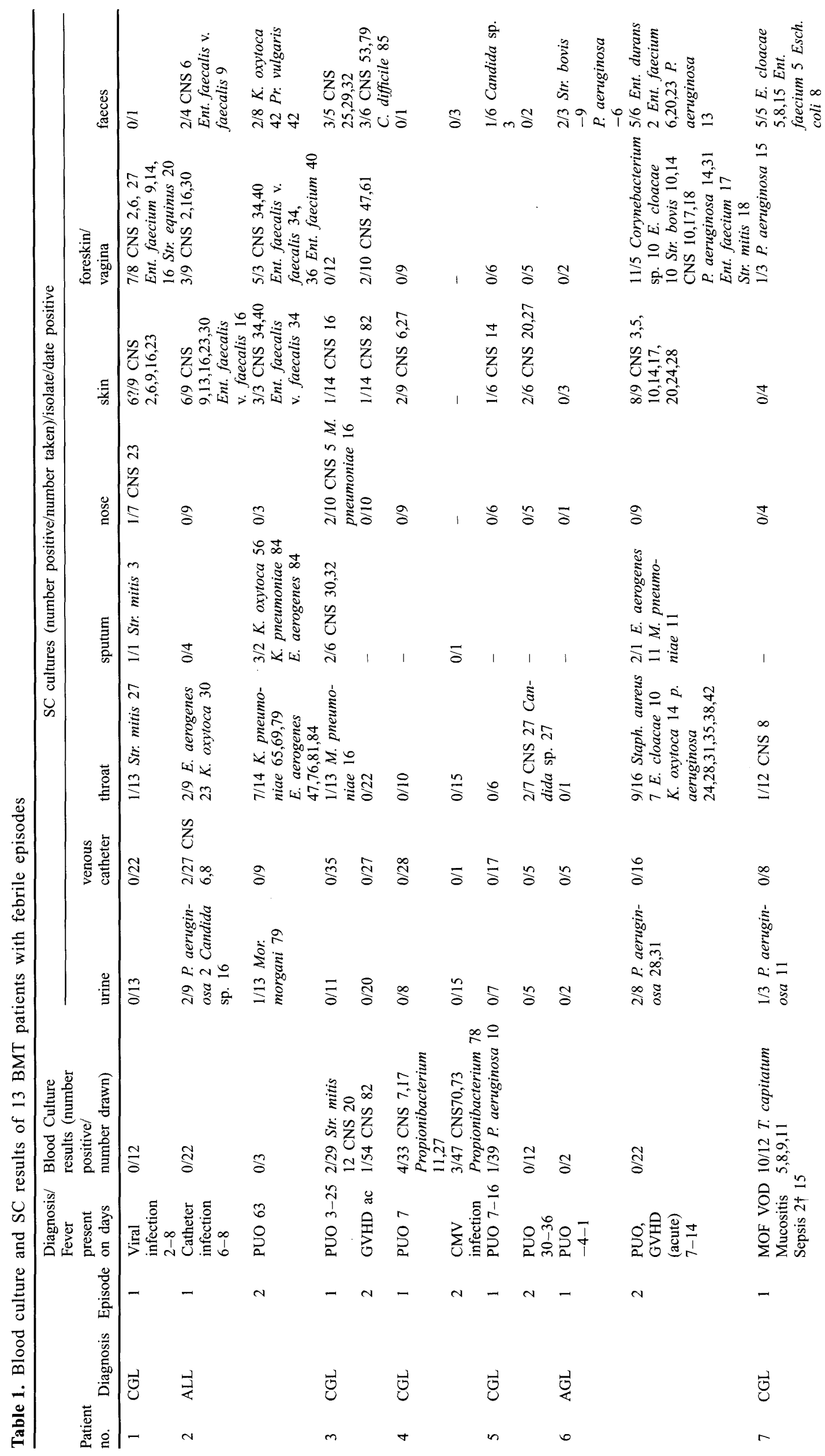




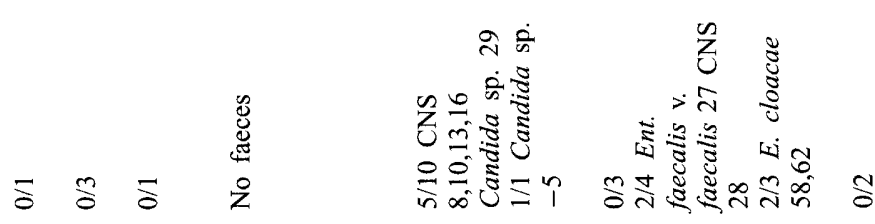

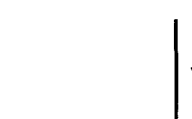

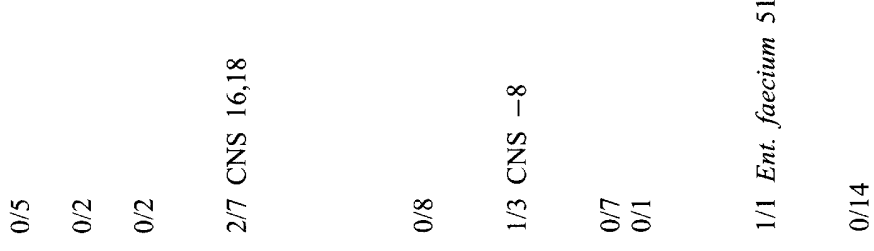<smiles>[CH]1C=C[CH-]1</smiles>

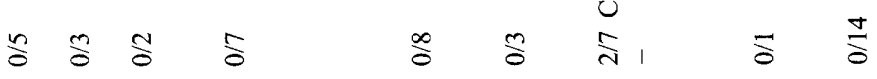

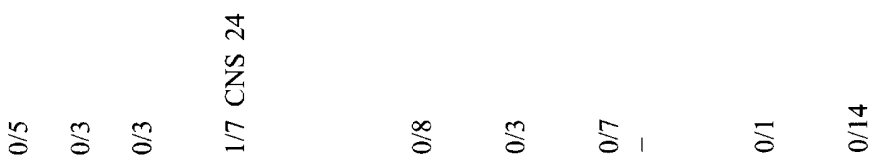

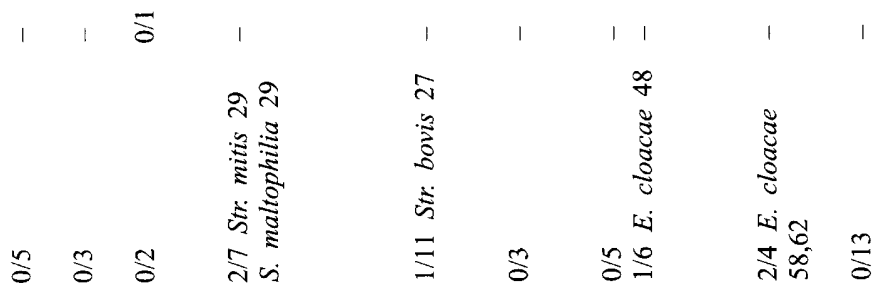

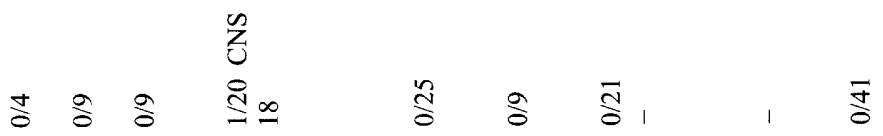

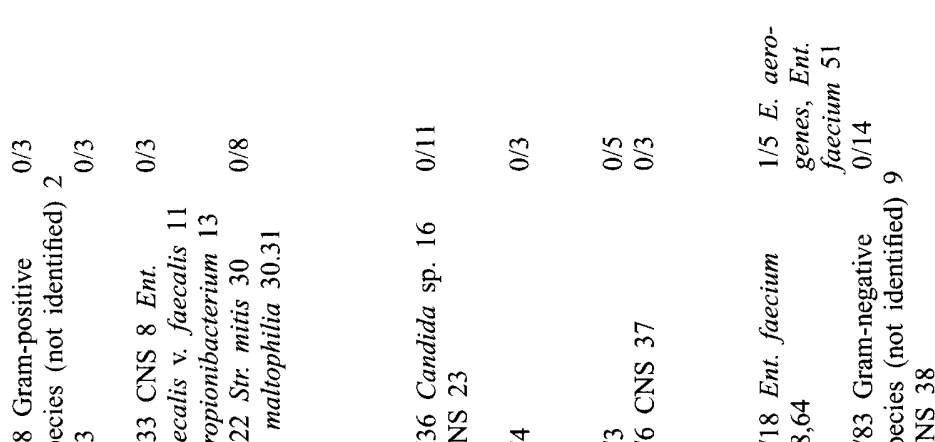

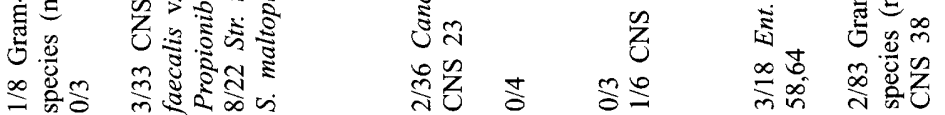

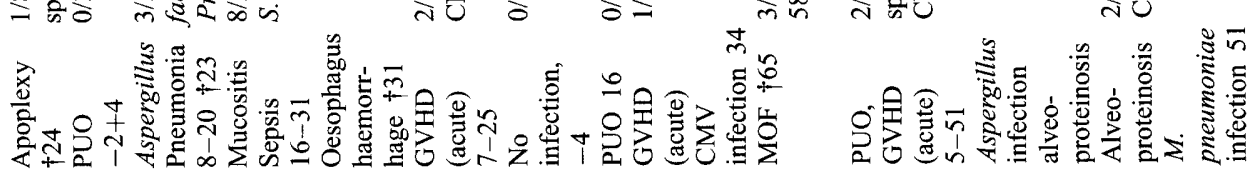

$n-4-$

Ð

$\overrightarrow{0}$

导

긍

$\therefore \quad=\simeq$

$\underline{2}$

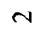


Table 2. Bacteria and fungi isolated from SC

\begin{tabular}{|c|c|c|c|c|c|c|}
\hline \multirow[b]{2}{*}{ Organism } & \multicolumn{5}{|c|}{ Time in weeks } & \multirow[b]{2}{*}{ Tota } \\
\hline & 1 & 2 & 3 & 4 & $5-8$ & \\
\hline \multicolumn{7}{|l|}{ Urinary samples $(175)$} \\
\hline Ent. faecium & & & & & 1 & 1 \\
\hline P. aeruginosa & 1 & 1 & & 1 & 1 & 4 \\
\hline Mor morgani & & & & & 1 & 1 \\
\hline E. aerogenes & & & & & 1 & 1 \\
\hline \multicolumn{7}{|c|}{ Venous catheter samples (338) } \\
\hline $\mathrm{CNS}$ & 2 & & 1 & & & 3 \\
\hline \multicolumn{7}{|l|}{ Throat samples (192) } \\
\hline $\mathrm{CNS}$ & & 1 & & 1 & & 2 \\
\hline Str. mitis & & & & 1 & 1 & 2 \\
\hline Str. bovis & & & & 1 & & 1 \\
\hline Staph. aureus & 1 & & & & & 1 \\
\hline K. pneumoniae & & & & & 3 & 3 \\
\hline K. oxytoca & & 1 & & & 1 & 2 \\
\hline E. aerogenes & & & & 1 & 4 & 5 \\
\hline E. cloacae & & 1 & & & 3 & 4 \\
\hline P. aeruginosa & & & & 2 & 4 & 6 \\
\hline S. maltophilia & & & & & 1 & 1 \\
\hline M. pneumoniae & & & 1 & & & 1 \\
\hline Candida spp. & & & & 1 & & 1 \\
\hline \multicolumn{7}{|l|}{ Sputum (16) } \\
\hline $\mathrm{CNS}$ & & & & 2 & & 2 \\
\hline Str. mitis & 1 & & & & & 1 \\
\hline K. oxytoca & & & & & 1 & 1 \\
\hline K. pneumoniae & & & & & 1 & 1 \\
\hline E. aerogenes & & 1 & & & 1 & 2 \\
\hline M. pneumoniae & & 1 & & & & 1 \\
\hline \multicolumn{7}{|l|}{ Nose samples (124) } \\
\hline CNS & 2 & & 1 & & & 3 \\
\hline M. pneumoniae & & & 1 & & & 1 \\
\hline \multicolumn{7}{|l|}{ Skin samples (136) } \\
\hline CNS & 5 & 6 & 7 & 7 & 4 & 29 \\
\hline Ent. faecalis v. faecalis & & & 1 & & 1 & , \\
\hline \multicolumn{7}{|c|}{ Foreskin or vagina samples (126) } \\
\hline CNS & 3 & 1 & 5 & 1 & 5 & 15 \\
\hline Ent. faecium & & 2 & 2 & & 2 & 6 \\
\hline Ent. faec. v. faec & & & & & 2 & 2 \\
\hline Str. equinus & & & 1 & & & 1 \\
\hline Str. bovis & & 2 & & & & 2 \\
\hline Str. mitis & & & 1 & & & 1 \\
\hline Corynebacterium sp. & & 1 & & & & 1 \\
\hline E. cloacae & & 1 & & & & 1 \\
\hline P. aeruginosa & & 1 & 1 & & 1 & 3 \\
\hline \multicolumn{7}{|l|}{ Faeces samples $(80)$} \\
\hline CNS & 1 & 3 & 1 & 2 & 4 & 11 \\
\hline Ent. durans & 1 & & & & & 1 \\
\hline Ent. faecium & 2 & & 2 & & & 4 \\
\hline Ent. faecalis v. faecalis & & 1 & & 1 & & 2 \\
\hline Str. bovis & & & & & 1 & 1 \\
\hline K. oxytoca & & & & & 1 & 1 \\
\hline E. cloacae & 1 & 1 & 1 & & 2 & 5 \\
\hline Pr. vulgaris & & & & & 1 & 1 \\
\hline Esch. coli & & 1 & & & & 1 \\
\hline P. aeruginosa & & 1 & & & & 1 \\
\hline C. difficile & & & & & 1 & 1 \\
\hline Candida spp. & 1 & & & & 1 & 2 \\
\hline
\end{tabular}

Species isolated (Tables 1 and 2): Acinetobacter calcoaceticus, Aspergillus sp. Candida spp., Clostridium difficile, Corynebacterium spp., Enterobacter aerogenes, E. cloacae, Enterococcus durans, Ent. faecalis var faecalis, Ent. faecium, Escherichia coli, Klebsiella oxytoca, $K$. pneumoniae, Morganella morgani, Mycoplasma pneumoniae, Propionibacterium spp., Proteus vulgaris, Pseudomonas aeruginosa, Staphylococcus aureus, coagulase-negative staphylococci, Stenotrophomonas maltophilia, Streptococcus bovis, Str. equinus, Str. mitis, Torulopsis capitatum. numbers instead of the normal flora (data not shown). This was probably due to the fact BMT patients spent weeks or months in hospital where they were exposed to heavy antimicrobial treatment. The intestinal flora of these patients contained Klebsiella spp., Enterobacter spp. and enterococci rather than Esch. coli even 1-3 months before and after transplantation. These conditions also led to the frequent isolation of C. difficile from these patients.

These data correspond to those of many authors [4,11-14] namely that CNS can be important pathogens in patients receiving immunosuppression therapy. The patients in this study were also infected by CNS and infections have been linked to the use of long-term indwelling venous catheters. However, the portal of entry of these organisms could also be the nose and throat and less commonly the bowel. Most infections arise from organisms in the upper respiratory or gastrointestinal tracts, but skin is of increasing importance [10]. Damage to the skin or mucosa by cytotoxic therapy or insertion of vascular access devices creates a portal of entry for microbes.

The findings that oral flora changed during illness and that gram-negative bacteria tend to replace streptococci are in accordance with the findings of Daw et al. [3] who found the mouth to be a reservoir of the former organisms. These results show that the reappearance of Enterobacteriaceae (Klebsiella and Enterobacter spp.) in the gut paralleled their appearance in the mouth. Accordingly, oral flora may serve as a monitor to indicate the re-appearance of the gut flora. Colonised patients endanger the intensive care unit (ICU) and hospital environments by acting as reservoirs of potential pathogens [14-16]; in particular, the presence of facultatively pathogenic microorganisms in the gut ( $P$. aeruginosa, $C$. difficile, etc.) carries increased risk for these patients.

Patients with positive SC and clinical signs of infection should be treated with combined and broad-spectrum antimicrobial agents that are not solely directed to the colonising organisms. Efforts should be made to determine specific treatment for clinically and microbiologically documented infections. To this end, close contact between physicians, infectious disease physicians and microbiologists, suitably sampled clinical material, and cautious considerations are essential. Only thus will the hoped-for results be achieved and the patient's life saved.

We thank G. Petrás of the Hospital Epidemiological Department of the B. Johan Institute of Public Health for critical reading of the manuscript.

\section{References}

1. Sinnott JT, Rubin RH. Infections in transplantation. In: Reese RE, Betts RF (eds) A practical approach to infectious diseases, 
3rd edn. An MSD handbook. Boston, Little, Brown \& Co 1991: 619-642.

2. Atkinson $\mathrm{K}$. (ed) Clinical bone marrow transplantation: a reference textbook. Cambridge, Cambridge University Press. 1994

3. Daw MA, MacMahon E, Keane CT. Surveillance cultures in the neutropenic patient. $J$ Hosp Infect 1988; 12: 251-261

4. De Jong PJ, De Jong MD, Kuijper EJ, van Der Lelie H. The value of surveillance cultures in neutropenic patients receiving selective intestinal decontamination. Scand J Infect Dis 1933 25: $107-113$

5. Schimpff SC. Surveillance cultures. $J$ Infect Dis 1981; 144: $81-84$.

6. Buckner CD, Clift RA, Sanders JE et al. Protective environment for marrow transplant recipients: a prospective study. $A n n$ Intern Med 1978; 89: 893-901.

7. Lányi B. Járványügyi és klinikai bakteriológia. Budapest, OKI 1980.

8. Marcus RE, Goldman JM. Management of infection in the neturopenic patient. $B M J$ 1986; 293: 406-408.

9. Kramer BS, Pizzo PA, Robichaud KJ, Witesbsky F, Wesley R. Role of serial microbiological surveillance and clinical evaluation in the management of cancer patients with fever and granulocytopenia. Am J Med 1982; 72: 561-568.
10. Milligan DW, Taylor M. Management of infection in the neutropenic patient. $B M J 1986 ; 293: 817$.

11. Winston DJ, Dudnick DV, Chapin M, Ho WG, Gale RP, Martin WJ. Coagulase-negative staphylococcal bacteremia in patients receiving immunosuppressive therapy. Arch Intern Med 1983; 143: $32-36$.

12. Clasener HAL, Vollaard EJ, van Saene HKF. Long-term prophylaxis of infection by selective decontamination in leukopenia and in mechanical ventilation. Rev Infect Dis 1987; 9: 295-328.

13. Rogers TRF. Infection associated with immunodeficiency. In: Parker MT, Collier L (eds) Topley and Wilson's: Principles of bacteriology, virology and immunity, 8th edn, vol 3. London, Arnold. 1990: 175-196.

14. Tetteroo GWM, Wagenvoort JHT, Bruining HA. Bacteriology of selective decontamination: efficacy and rebound colonization. J Antimicrob Chemother 1994; 34: 139-148.

15. Massanari RM, Hierzhol WJ. The intensive care unit. In: Bennett JE, Brachman PS (eds) Hospital infections, 2nd edn. Boston Little, Brown \& Co. 1986: 285-297.

16. Selden R, Lee S, Wang WLL, Bennett J, Eickhoff TC. Nosocomial Klebsiella infections: intestinal colonization as a reservoir. Ann Intern Med 1971; 74: 657-664. 